\title{
Crp of Streptomyces coelicolor is the third transcription factor of the large CRP-FNR superfamily able to bind cAMP
}

\author{
Adeline Derouaux ${ }^{\mathrm{a}}$, Dominique Dehareng ${ }^{\mathrm{a}}$, Elke Lecocq ${ }^{\mathrm{b}}$, Serkan Halici ${ }^{\mathrm{c}}$, \\ Harald Nothaft ${ }^{\mathrm{c}}$, Fabrizio Giannotta ${ }^{\mathrm{a}}$, Georgios Moutzourelis ${ }^{\mathrm{a}}$, Jean Dusart ${ }^{\mathrm{a}}$, \\ Bart Devreese $^{\mathrm{b}}$, Fritz Titgemeyer ${ }^{\mathrm{c}}$, Jozef Van Beeumen ${ }^{\mathrm{b}}$, Sébastien Rigalia,* \\ a Centre d'Ingénierie des Protéines, Université de Liège, Institut de Chimie B6a, B-4000, Liège, Belgium \\ ${ }^{\mathrm{b}}$ Laboratory for Protein Biochemistry and Protein Engineering, Ghent University, K.L. Ledeganckstraat 35, 9000 Ghent, Belgium \\ ${ }^{\mathrm{c}}$ Lehrstuhl für Mikrobiologie, Friedrich-Alexander-Universität Erlangen-Nürnberg, Staudtstrasse 5, 91058 Erlangen, Germany
}

Received 4 October 2004

Available online 6 November 2004

\begin{abstract}
The chromosomal inactivation of the unique transcription factor of Streptomyces coelicolor that displays a cyclic-nucleotidebinding domain, $\mathrm{Crp}^{\mathrm{Sco}}$, led to a germination-defective phenotype similar to the mutant of the adenylate cyclase gene (cya) unable to produce cAMP. By means of cAMP affinity chromatography we demonstrate the specific cAMP-binding ability of Crp ${ }^{\text {Sco }}$, which definitely demonstrate that a Cya/cAMP/Crp system is used to trigger germination in S. coelicolor. However, electromobility shift assays with the purified $\mathrm{Crp}^{\mathrm{Sco}}$-CAMP complex and the CRP-like cis-acting element of its own promoter failed. Moreover, we were unable to complement an Escherichia coli crp mutant in trans with $\mathrm{Crp}^{\mathrm{Sco}}$. The fact that $\mathrm{Vfr}$ from Pseudomonas aeruginosa and GlxR from Corynebacterium glutamicum could complement such an E. coli mutant suggests that the way Crp ${ }^{\text {Sco }}$ interacts with DNA should mechanistically differ from its most similar members. This hypothesis was further supported by homology modelling of $\mathrm{Crp}^{\mathrm{Sco}}$ that confirmed an unusual organisation of the DNA-binding domain compared to the situation observed in $\mathrm{Crp}^{\mathrm{Eco}}$.

(C) 2004 Elsevier Inc. All rights reserved.
\end{abstract}

Keywords: cAMP; Germination; CRP-FNR family; Cyclic-nucleotide-binding domain; Streptomyces

Streptomycetes are Gram-positive soil bacteria with a complex life cycle that includes germination, vegetative mycelial growth, aerial mycelium formation, and spore morphogenesis [1]. In order to restore vegetative growth once conditions become favourable, bacterial spores must be able to sense environmental changes that trigger the germination process. In the initial stages of germination, undefined signals trigger influx of water into spores, resulting in an increase in size and decreased phase brightness followed by germ-tube emergence [2]. Recently, we focused on the study of the unique

\footnotetext{
${ }^{*}$ Corresponding author. Fax: +3243663364 .

E-mail address: srigali@ulg.ac.be (S. Rigali).
}

CRP-FNR member of Streptomyces coelicolor, encoded by orf SCO3571 or the $\mathrm{crp}$ gene (noted $\mathrm{Crp}^{\mathrm{Sco}}$ protein) [3]. Its chromosomal inactivation led to a germinationdefective mutant of which the phenotype is similar to the mutant of the adenylate cyclase gene (cya) unable to produce cAMP [3,4]. These similar phenotypes for both mutants and the observation of a peak of cAMP accumulation during germination [4] suggest that cAMP should be regarded as a molecular signal that initiates germination events. However, before concluding that a $\mathrm{Cya} / \mathrm{cAMP} / \mathrm{Crp}^{\mathrm{Sco}}$ system is used to trigger germination in $S$. coelicolor, clear evidence for an interaction of $\mathrm{Crp}^{\mathrm{Sco}}$ with cAMP still needs to be demonstrated. Furthermore, the cAMP-binding ability of $\mathrm{Crp}^{\mathrm{Sco}}$ became somehow more doubtful as a recent phylogenetic 
analysis of CRP-FNR members classified this transcription factor (TF) into the heme-containing CooA subfamily [5]. This subclassification suggests that the molecular event responsible for the DNA binding of the transcription factor is the binding of a carbon monoxide (CO) molecule via a prosthetic group [5].

We report here a refined in silico analysis that strongly suggests the classification of $\mathrm{Crp}^{\text {Sco }}$ into the cAMP-binding subfamily. We provide experimental proof for this by in vitro-binding assays and by isolation of cAMP-binding proteins in vivo. We further show that $\mathrm{Crp}^{\mathrm{Sco}}$ does not bind to a suspected cis-acting element suggesting that the mechanism of $\mathrm{Crp}^{\mathrm{Sco}}$ function must differ from its most similar homologues. These data are supported by homology modelling and discussed in detail.

\section{Materials and methods}

Bacterial strains, culture conditions, and preparation crude extracts. Escherichia coli strains XL1 blue, and BL21(DE3), were grown in liquid or solid LB medium at $37^{\circ} \mathrm{C}$. The $E$. coli crp mutant LDN-3 $\Delta \mathrm{crp}-45 \mathrm{Spc}^{\mathrm{r}}$, which has been constructed by P1-transduction of the $\Delta$ crp-45 deletion from a CA-8404 derivative into JWL184-1, was used for complementation assays [6,7]. S. coelicolor A3(2) M145 (SCP1 ${ }^{-}$ $\mathrm{SCP} 2^{-}$prototroph) and Streptomyces lividans TK24 were grown in YEME liquid medium [1].

Expression and purification of His-tagged $\mathrm{Crp}^{\mathrm{Sco}}$ in E. coli. The crp gene of $S$. coelicolor was amplified from genomic DNA by PCR with $P f u$ DNA polymerase. The oligonucleotides used for crp amplification were 5'-CATATGGACGACGTTCTGCGGCGCAA-3' and 5'TTAAAGCTTGCGGGAGCGCTTGGCCAGTC-3' (restriction sites underlined). The PCR product was cloned into pGEM-T-Easy and sequenced. The resulting plasmid, pCIP261, was digested with $N d e$ I and HindIII, and the isolated $675 \mathrm{bp} c r p$ fragment was ligated with the E. coli expression vector pET22b. The resulting plasmid, pCIP262, which encodes a Crp ${ }^{\text {Sco }}$ protein with a C-terminal hexahistidine tag $\left(\mathrm{Crp}^{\mathrm{Sco}}-\mathrm{His}_{6}\right)$, was transformed into $E$. coli BL21(DE3). Expression was induced with $1 \mathrm{mM}$ IPTG after growth to an $A_{600}$ of 0.6 in $500 \mathrm{ml} \mathrm{LB}$ medium at $37^{\circ} \mathrm{C}$. Incubation was continued for $3 \mathrm{~h}$ and cells were harvested by centrifugation and resuspended in $200 \mathrm{ml}$ of $100 \mathrm{mM}$ sodium phosphate buffer ( $\mathrm{pH} 7.0$ ) containing $250 \mathrm{mM} \mathrm{NaCl}$. Cells were disrupted with a French press and cell debris was subsequently removed by centrifugation. Benzonase was added to the supernatant ( $1 \mu \mathrm{l}$ for $100 \mathrm{ml}$ of supernatant) and left at $4{ }^{\circ} \mathrm{C}$ overnight. $\mathrm{Crp}^{\mathrm{Sco}}{ }^{-} \mathrm{His}_{6}$ was purified from soluble cell extract on a nickel-nitrilotriacetic acid $\left(\mathrm{Ni}^{2+}-\mathrm{NTA}\right)$ chelating column. $\mathrm{Crp}^{\mathrm{Sco}}-\mathrm{His}_{6}$ eluted mainly at $250 \mathrm{mM}$ imidazole from the column.

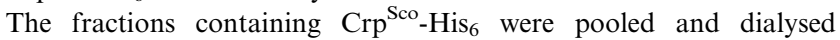
overnight against $100 \mathrm{mM}$ sodium phosphate buffer ( $\mathrm{pH}$ 7.0) containing $250 \mathrm{mM} \mathrm{NaCl}$.

Expression and purification of His-tagged $X \ln R$ in E. coli. The $x \ln R$ gene of Streptomyces sp. EC3 was amplified from genomic DNA by PCR with $P f u$ DNA polymerase. The oligonucleotides used were $5^{\prime}$ GGGTTAGCATATGCCTGCTACCGACGACCGTCGGCCCAA GTACC-3' and 5'-CCGCTAAGCTTGGCCGGGTCTCCGGGCG CTGTGGCGTCGG-3' (restriction sites underlined). Cloning procedures, sequencing, and overexpressing vectors used, and production and purification conditions were as described above for Crp ${ }^{\text {Sco }}$ with the following differences: maximal production of $\mathrm{X} \ln \mathrm{R}$ was obtained after an overnight induction with IPTG, and XlnR-His 6 eluted mainly at $120 \mathrm{mM}$ imidazole.
Anti-Crp $p^{\text {Sco }}$ antibodies and Western blot analysis. A polyclonal anti-Crp $^{\text {Sco }}$ antiserum was generated by immunizing New Zealand white rabbits with purified $\mathrm{Crp}^{\text {Sco }}{ }_{-\mathrm{His}_{6}}$. The crude serum was used in immunoblotting at a final dilution of 1:3000. Twenty-five micrograms of total cytoplasmic proteins was separated by SDS-PAGE $(12 \%)$ and electroblotted onto a nitrocellulose membrane. Immunoblot detection of anti-Crp ${ }^{\text {Sco }}$ antibodies was carried out with goat alkaline phosphatase-conjugated anti-rabbit antibodies, in combination with a color reaction of 5-bromo-4-chloro-3-indoyl phosphate and nitroblue tetrazolium (Bio-Rad; immunoblot alkaline phosphate assay system).

cAMP-binding assay. cAMP was from Acros Organics. cAMP affinity matrix was purchased from Sigma. cAMP was immobilised on beaded agarose via the $\mathrm{C} 8$ residue of the base, with a spacer of 9 atoms. An equimolar quantity of purified $\mathrm{Crp}^{\mathrm{Sco}}-\mathrm{His}_{6}$ was incubated in batch with $200 \mu \mathrm{l}$ of C-8 cAMP-agarose, previously equilibrated in buffer $\mathrm{A}$ $(100 \mathrm{mM}$ sodium phosphate buffer, $\mathrm{pH} 7,50 \mathrm{mM} \mathrm{NaCl})$, at $25^{\circ} \mathrm{C}$ during $30 \mathrm{~min}$ on a rotary mixer. After washing 8 times with $500 \mu \mathrm{l}$ buffer $\mathrm{A}$, bound proteins were eluted in two times by $30 \mathrm{~min}$ incubation in $500 \mu \mathrm{l}$ buffer A supplemented with cAMP $30 \mathrm{mM}$ at $25^{\circ} \mathrm{C}$. Fractions were analysed by $12 \%$ SDS-PAGE. For the cAMP-binding assay with soluble protein extract of $S$. coelicolor, the salt concentration of the buffer A was increased to $150 \mathrm{mM} \mathrm{NaCl}$ (buffer B) to diminish nonspecific interactions between proteins and the matrix. We used $300 \mu \mathrm{l}$ cAMP-agarose incubated 2 times with $750 \mu \mathrm{l}$ S. coelicolor protein extract $(6 \mathrm{mg} / \mathrm{ml})$ from a 2 day culture in YEME. Determination of protein concentrations was performed using the 2-bicinchoninic acid assay (BCA protein assay; Pierce).

Tryptic digest and MS characterisation. The affinity purified proteins were separated by SDS-PAGE and stained with Coomassie blue $\mathrm{R} 250$. The identification of proteins was performed as described previously by Vanrobaeys et al. [8].

Bioinformatics and molecular modelling. The amino acids sequences of the CRP-FNR members were obtained using the Sequence Retrieval System (SRS) available at the Expert Protein Analysis System (ExPASy) Molecular Biology Server: http://www.expasy.org/, Expasy Home page. Multiple alignments were developed with MultiAlin [9] (available at http://prodes.toulouse.inra.fr/multalin).

The 3D model of $\mathrm{Crp}^{\text {Sco }}$ was built with the program ESyPred3D [10] based on homology and comparative modelling, using Crp ${ }^{\mathrm{Eco}}$ (1I5Z PDB code) as reference. The $\mathrm{Crp}^{\mathrm{Sco}}$ residue D15 was superimposed to the $\mathrm{Crp}^{\mathrm{Eco}} \mathrm{T} 7$ (Fig. 1). One deletion and one insertion were detected in order to match at best the two sequences. The deletion occurred in the turn from P86 to P88, superimposed to the Crp ${ }^{\mathrm{Eco}}$ turn from E78 to E81. The insertion lay in the turn from G168 to V172 related to $\mathrm{Crp}^{\mathrm{Eco}}$ residues from D161 to Q164. The subsequent geometry optimisation was performed on a dimer including two molecules of cAMP and water molecules. The initial conformation was built mostly like the dimer structure $1 \mathrm{I} 5 \mathrm{Z}$ of $\mathrm{Crp}^{\mathrm{Eco}}$. Both $\mathrm{Crp}^{\mathrm{Sco}}$ and $\mathrm{Crp}^{\mathrm{Eco}}$ dimers containing two cAMP and crystallisation water molecules were optimised at the molecular mechanics level (MM) in order to compare equivalently resulting structures. The optimisation was performed at the MM level using the program Discover [11] with the Amber force field $[12,13]$ and a relative dielectric constant equal to 2 .

Overexpression and purification of Crp ${ }^{\text {Sco }}$ in Streptomyces lividans $T K 24$. The crp gene of $S$. coelicolor was amplified from pCIP261 with $P f u$ DNA polymerase and oligonucleotides $5^{\prime}$-CCAGATCTGTGGAC GACGTTCTGCGGCGC-3' and $5^{\prime}$-CTGCAGTCAGCGGGAGCG CTTGGCCAGTCG-3' (restriction sites underlined). The PCR product was cloned into pGEM-T-Easy and sequenced. The resulting plasmid pCIP266 was digested with Bg/II and PstI, and the isolated 675 bp crp fragment was ligated with the Vpro p145.10 vector [14] digested by the same restriction enzymes, giving rise to pCIP267. Protoplasts of $S$. lividans TK24 were transformed by pCIP267. SDSPAGE revealed that the production was maximal after $90 \mathrm{~h}$ of growth at $28{ }^{\circ} \mathrm{C}$. Mycelium from a $100 \mathrm{ml}$ culture was pelleted, washed, resuspended in $4 \mathrm{ml}$ of $20 \mathrm{mM}$ Tris- $\mathrm{HCl}, \mathrm{pH} \mathrm{7.5}$, and disrupted by 


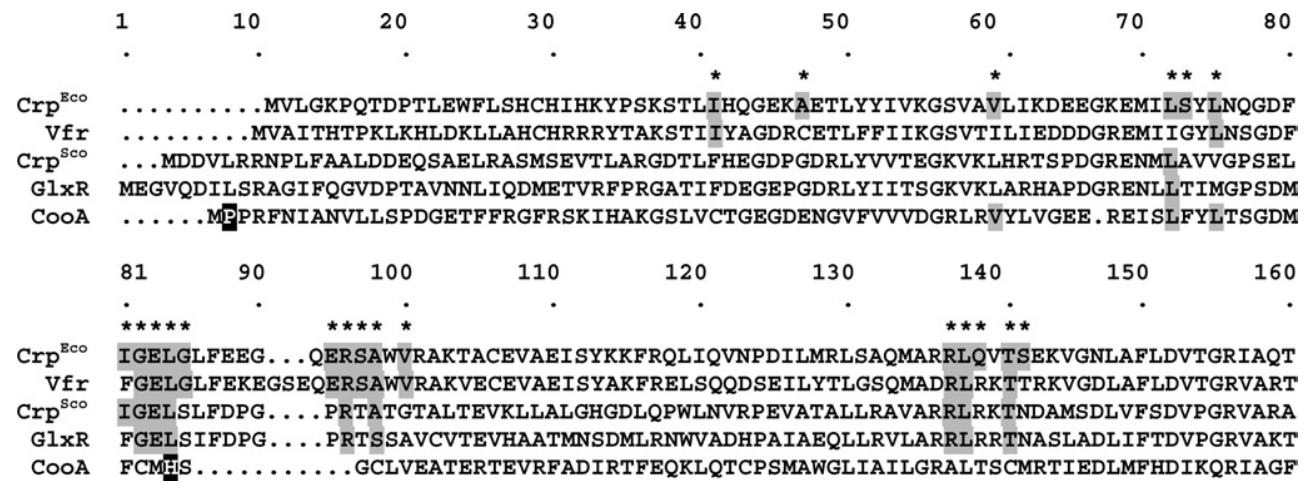

Fig. 1. Positional conservation of amino acids involved in cAMP binding. The multiple alignment of the various effector-binding domains was constructed with MultiAlin. Crp ${ }^{\text {Eco }}$, cAMP receptor protein of E. coli; Vfr, virulence factor regulator of Pseudomonas aeruginosa; Crp ${ }^{\text {Sco }}$, cAMP receptor protein of Streptomyces coelicolor; GlxR, regulator of glyoxylate bypass of C. glutamicum; and CooA, CO-dependent transcriptional activator of Rhodospirillum rubrum. Positional identities of residues involved in cAMP binding in Crp ${ }^{\text {Eco }}$ (marked with asterisks) are highlighted in grey. Residues involved in heme binding in CooA are highlighted in black.

sonication in a Branson ultrasonic disintegrator at a $12 \mu \mathrm{m}$ amplitude for three 30 -s bursts. The soluble cell fraction was obtained by centrifugation $(16,000 \mathrm{~g}, 30 \mathrm{~min})$ of the lysate. Being genetically very close to $S$. coelicolor, one should also expect that some proteins of $S$. lividans TK24 interact with the cAMP matrix as observed previously. In order to avoid the purification of these proteins, we added ion exchange chromatography on a HiTrap QHP column $(5 \mathrm{ml})$ as a first step of purification before the cAMP affinity chromatography step. The column was equilibrated with $20 \mathrm{mM}$ Tris- $\mathrm{HCl}, \mathrm{pH} 7.5$. The adsorbed $\mathrm{Crp}^{\mathrm{Sco}}$ was eluted with a linear $\mathrm{NaCl}$ gradient $(50 \mathrm{ml})$ from 0.3 to $0.75 \mathrm{M}$. The fractions were analysed by $15 \%$ SDS-PAGE. Fractions containing $\mathrm{Crp}^{\mathrm{Sco}}$ were pooled and dialysed overnight against buffer $\mathrm{B}$ (for buffer composition, see the cAMP-binding assay procedure). The protein was further purified on a cAMP-agarose column of $5 \mathrm{ml}$. The column was equilibrated with buffer B. The fractions were loaded on the column. The column was washed with $40 \mathrm{ml}$ buffer B and the adsorbed $\mathrm{Crp}^{\mathrm{Sco}}$ was eluted with $15 \mathrm{ml}$ buffer B supplemented with $30 \mathrm{mM}$ cAMP. The fractions were analysed by $15 \%$ SDS-PAGE. About $2 \mathrm{mg}$ of nearly pure $(>95 \%) \mathrm{Crp}^{\mathrm{Sco}}$ was obtained from a $100 \mathrm{ml}$ culture. For gel shift assays we used fractions of $\mathrm{Crp}^{\mathrm{Sco}}$ eluted from the cAMP-agarose column with $1 \mathrm{M} \mathrm{NaCl}$ in order to obtain the TF not complexed to its ligand. The fractions containing $\mathrm{Crp}^{\mathrm{Sco}}$ were pooled and dialysed overnight against $100 \mathrm{mM}$ sodium phosphate buffer $(\mathrm{pH}$ 7.0) containing $50 \mathrm{mM} \mathrm{NaCl}$.

Electromobility gel shift assays. Electromobility gel shift assays (EMSA) was performed as described previously $[3,15]$. The fluorescent double-stranded oligonucleotide representing the cis-acting element upstream $\operatorname{crp}$ (5'-Cy5-CATCCTTGTGACAGATCACACTGTTT-3') was used as the DNA probe (underlined nucleotides represent the signature recognised by CRP-like members).

E. coli crp mutant complementation assays. The crp genes of $S$. coelicolor and $E$. coli were cloned behind the constitutive lac $Z$ promoter into the pSU2718 derivative $\mathrm{pF} 76$ by replacing the $A f \mathrm{III}-\mathrm{Hin}$ dIII fragment with the respective $c r p$ fragment, giving pFT171 $\left(c r p^{\mathrm{Sco}+}\right)$ and pFT172 $\left(c r p^{\mathrm{Eco}+}\right)$. The $c r p$ genes were amplified by PCR from chromosomal DNA of $S$. coelicolor A3(2) and E. coli DH5a using appropriate oligonucleotides (crpScol, AGTACACGTGGAC GACGTTCTGCGGCGCAACC; crpSco2, ATATAAGCTTGGTCA GCGGGAGCGCTTGGC; crpEco1, GTACACGTGGTGCTTGG CAAACCGC; crpEco2, ATATAAGCTTGATTAACGAGTGCCG TAAACG; restriction sites are underlined). Plasmids pFT76 (control), pFT171, and pFT172 were each transformed into the E. coli crp deletion mutant LDN-3 and analysed on MacConkey agar plates supplemented with either $25 \mathrm{mM}$ lactose or maltose for their capacity to restore sugar fermentation. E. coli $\mathrm{DH} 5 \alpha$ was used as wild type control.

\section{Results and discussion}

\section{Comparative study of amino acids involved in cAMP binding}

Crp of E. coli $\left(\mathrm{Crp}^{\mathrm{Eco}}\right)$ and virulence factor regulator (Vfr) of Pseudomonas aeruginosa are actually the only two members of the large CRP-FNR superfamily that exhibit a cAMP-binding ability $[16,17]$. We checked whether residues involved in cAMP binding in $\mathrm{Crp}^{\mathrm{Eco}}$ and $\mathrm{Vfr}$ were also present in $\mathrm{Crp}^{\text {Sco }}$ (Fig. 1). Crp ${ }^{\mathrm{Eco}}$ and Vfr share $67 \%$ of positional identity, and $\mathrm{Crp}^{\text {Sco }}$ displays $48 \%$ identity with both of them. In contrast, in CooA only $24 \%$, $19 \%$, and $14 \%$ of the analysed residues are identical to $\mathrm{Crp}^{\mathrm{Eco}}$, Vfr, and $\mathrm{Crp}^{\mathrm{Sco}}$, respectively. For other CRPFNR proteins not able to bind cAMP, the levels of positional identity were between $5 \%$ and $24 \%$ (data not shown), indicating that the level of conservation of residues involved in cAMP is much higher in $\mathrm{Crp}^{\mathrm{Sco}}$ than in all other representative members of the cAMP-non-binding subfamilies. Moreover, the residues involved in heme binding in CooA [18] are not present in $\mathrm{Crp}^{\mathrm{Sco}}$ (Fig. 1) which comforts the idea of an inappropriate clustering among CooA members [7]. We included GlxR of Corynebacterium glutamicum in our multiple alignment (Fig. 1), which also belongs to the CooA subgroup. Clear evidences for cAMP-GlxR interactions are still lacking but the involvement of cAMP as effector has been strongly suggested, as the binding of purified GlxR to its target promoter could be demonstrated only in the presence of cAMP [19]. These data support the feeling of Korner et al. [5] that the CooA subfamily is one of the less well defined and a relocation of members into other CRP-FNR subfamilies may be necessary.

\section{Modelisation of the cAMP-binding pocket}

We built the 3D model of $\mathrm{Crp}^{\text {Sco }}$ using $\mathrm{Crp}^{\text {Eco }}$ dimers containing two cAMP and crystallisation water 
molecules as reference. In $\mathrm{Crp}^{\mathrm{Eco}}$, seven direct interactions are found with cAMP: five between each cAMP and V49, E72, R82, S83, T127 of the monomer to which it is linked, and two with L124 and S128 of the other monomer. In $\mathrm{Crp}^{\mathrm{Sco}}$, seven direct interactions were also found: five from each monomer involving L57, L69, E80, R89 and the HN backbone of T90, and two with residues R132 and $\mathrm{N} 135$ of the second monomer (Fig. 2). The involvement of R132 of the second monomer in the linking of cAMP in $\mathrm{Crp}^{\mathrm{Sco}}$ should be responsible for a much stronger binding than the one resulting from L124 in Crp ${ }^{\mathrm{Eco}}$.

Indirect interactions were also predicted. In $\mathrm{Crp}^{\mathrm{Eco}}$, the residue Q125 of one monomer indirectly interacts with the cAMP of the other through a water molecule. The residue R123 is not in direct contact with cAMP but clearly orients E72. As for E81, it is oriented toward the solvent and does not seem to be directly related with cAMP. In $\mathrm{Crp}^{\mathrm{Sco}}$ an indirect contact with T134 occurs through a water molecule. R130 is linked with E80 through a salt bridge, like R132-E72 in Crp ${ }^{\text {Eco }}$. Thus, on the simple basis of the complex $\mathrm{Crp}^{\mathrm{Sco}}$-cAMP optimised geometry, the residues of the cAMP-binding

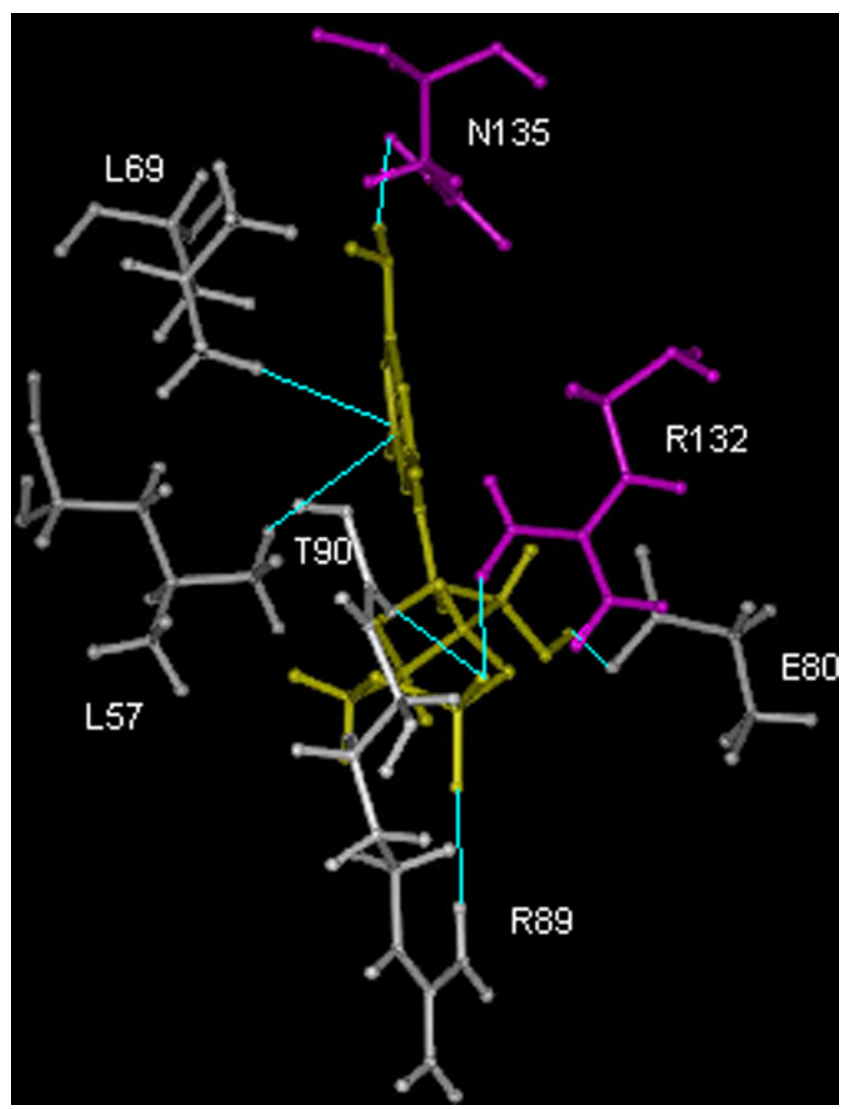

Fig. 2. Direct interactions within the cAMP-binding pocket of $\mathrm{Crp}^{\mathrm{Sco}}$. cAMP (yellow) surrounded by its direct residue environment (white for the first monomer, magenta for the second). (For interpretation of the references to color in this figure legend, the reader is referred to the web version of this paper). pocket should be capable of cAMP binding in a quite similar manner than the one observed in $\mathrm{Crp}^{\mathrm{Eco}}$.

\section{$\mathrm{Crp}^{\text {Sco }}$ is able to bind $\mathrm{cAMP}$}

To demonstrate the interaction of $\mathrm{Crp}^{\text {Sco }}$ with cAMP as suggested by the above-presented in silico data, we applied purified his-tagged $\mathrm{Crp}^{\mathrm{Sco}}\left(\mathrm{Crp}^{\mathrm{Sco}}-\mathrm{His}_{6}\right)$ to a cAMP-agarose matrix. The protein remained bound to the matrix until it was eluted with free cAMP

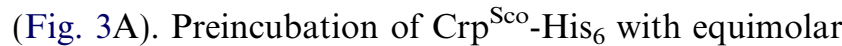
amounts of cAMP abolished binding to cAMP agarose, confirming a specific interaction via cAMP (Fig. 3B). As a negative control, we used the purified $X \ln R$ protein, a member of the GntR superfamily [20], for which no cyclic-nucleotide-binding properties have been reported so far. XlnR-His 6 was not retained by the cAMP matrix and was completely removed from the reaction mixture during the first wash step (Fig. 3C).

In a complementary approach, we were able to isolate four proteins from a crude $S$. coelicolor cell extract by cAMP affinity chromatography (Fig. 4A). The four proteins corresponded to molecular weights of approximately 20, 25, 37, and $40 \mathrm{kDa}$. Western blot analyses revealed that the $25 \mathrm{kDa}$ band was $\mathrm{Crp}^{\text {Sco }}$ (Fig. 4B), which indicates that cAMP binding to $\mathrm{Crp}^{\mathrm{Sco}}$ occurs in vivo. Using the same cAMP-binding assays, Brodbeck et al. [21] only isolated a nucleoside diphosphate (NDP) kinase from total extracts of $S$. coelicolor MT1110. We did not capture this protein, but the culture conditions were different. Moreover, Brodbeck et al. used a high salt concentration $(1 \mathrm{M} \mathrm{NaCl})$ in washes, so it is possible that the other proteins we captured were eluted during the washing steps. We confirmed this hypothesis as overexpressed $\mathrm{Crp}^{\text {Sco }}$ could be partially eluted from the cAMP affinity column by addition of $1 \mathrm{M} \mathrm{NaCl}$ (data not shown).

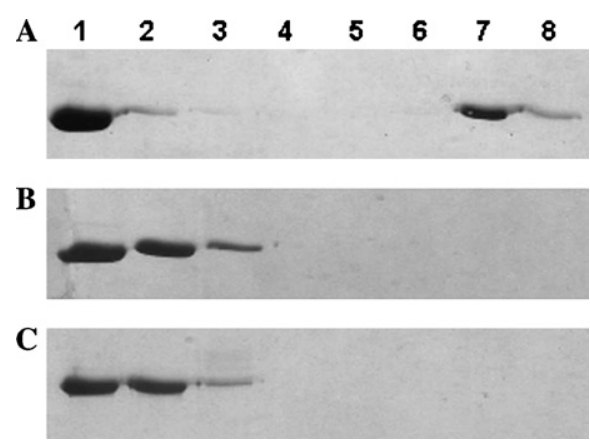

Fig. 3. The cAMP-binding ability of $\mathrm{Crp}^{\mathrm{Sco}}-\mathrm{His}_{6}$. Twelve percent

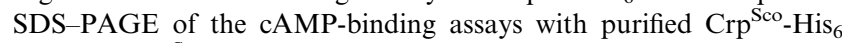
(A), with $\mathrm{Crp}^{\mathrm{Sco}}$-His ${ }_{6}$ pre-incubated with cAMP in equimolar quantity before the cAMP-binding assay (B), and as a negative control with purified $\mathrm{X} \operatorname{lnR}-\mathrm{His}_{6}(\mathrm{C})$. Lane 1, starting sample; lane 2, fraction unbounded to the cAMP matrix; lanes 3-6, fractions eluted during washing steps; and lanes 7 and 8 , fractions eluted with $30 \mathrm{mM}$ cAMP. 


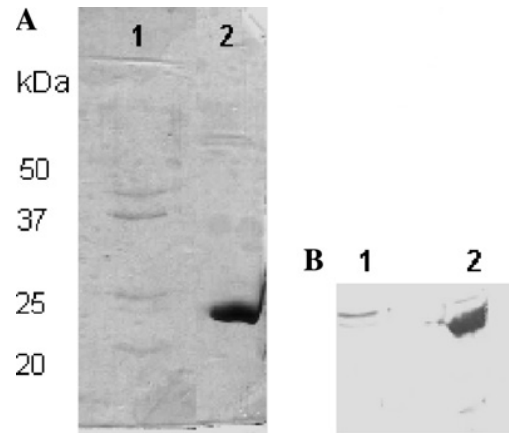

Fig. 4. Isolation of cAMP-binding proteins from the total soluble extract of $S$. coelicolor and identification of $\mathrm{Crp}^{\mathrm{Sco}}$. Twelve percent SDS-PAGE representing cAMP-binding proteins isolated from the crude extract of $S$. coelicolor (A) and Western blotting with $\mathrm{Crp}^{\mathrm{Sco}}$ antibodies (B). Fraction eluted with $30 \mathrm{mM}$ cAMP (lane 1) and purified $\mathrm{Crp}^{\mathrm{Sco}}-\mathrm{His}_{6}$ (lane 2).

\section{Identification of other cAMP-binding proteins in S. coelicolor}

Tryptic peptides of the three other putative cAMPbinding proteins isolated were analysed by mass spectroscopy. The identification of the $40 \mathrm{kDa}$ protein failed. The $20 \mathrm{kDa}$ protein was identified as ORF SCO3907, encoding a putative single-strand DNA-binding protein (SSB) of 19,906 Da, with a C-terminus that contains glycine-rich repeats. SSB proteins play essential roles in many processes related to DNA metabolism such as DNA replication, repair, and homologous genetic recombination. Blast search revealed $61 \%$ identity with mycobacterial SSB proteins [22]. The latter have clearly been shown to be able to interact with nucleic acids, and with proteins involved in DNA metabolism, through their C-terminal glycine-rich domain. As no specific interactions with cyclic-nucleotides have been reported so far, it is difficult to state whether the isolation of this protein was due to a direct binding with the cAMP matrix or via protein-protein interaction with one of the three other proteins isolated from the crude extracts.

Two proteins were identified from the $37 \mathrm{kDa}$ band: (i) SCO0741, which encodes a putative oxidoreductase with a molecular mass of $37,949 \mathrm{Da}$, and (ii) SCO1087, a putative aldolase with a molecular mass of $39,107 \mathrm{Da}$. SCO0741 displays $71 \%$ identity with mycobacterial AdhE2 proteins, and 70\% with $\mathrm{FadH}$, a NAD/mycothiol-dependent formaldehyde dehydrogenase of Amycolatopsis methanolica. Both proteins belong to the zinc-dependent alcohol dehydrogenase family. Proteins of this family use $\mathrm{NAD}^{+}$as coenzyme and two zinc ions as cofactors [23]. The capture of a $\mathrm{NAD}^{+}$-binding protein using a cAMP matrix was also reported previously by Laukens et al. [24] and Weber et al. [25], as they isolated a glyceraldehyde 3-phosphate dehydrogenase (GAPDH) from tobacco cells and from various mammalian tissue extracts, respectively. If a preincubation with $\mathrm{NAD}^{+}$was performed, GAPDH was not retained on the cAMP matrix [24]. Interaction of GAPDH with the matrix appears to be specific for the nucleotide moiety [25]. In addition, it was shown in yeast that cAMP is able to inhibit the GAPDH activity, but the inhibition appeared to be relatively weak [26]. It is suggested that GAPDH binds $\mathrm{NAD}^{+}$and cAMP at the same site [24-26] which could explain the capture of SCO0741 by the CAMP-agarose matrix.

Regarding the second protein identified into the $37 \mathrm{kDa}$ band, SCO1087 has an average $45 \%$ identity with L-threonine aldolase from many different bacterial strains [27]. These enzymes bind pyridoxal phosphate (PLP) as coenzyme. To our knowledge, this is the first time that a PLP-binding enzyme has been isolated using a cAMP matrix.

\section{DNA-binding ability of $\mathrm{Crp}^{\mathrm{Sco}}$}

Although the ligand-binding capability of $\mathrm{Crp}^{\mathrm{Sco}}$ has now been demonstrated, it still remains to be established whether the protein-cAMP interaction is necessary to confer DNA binding. We previously demonstrated, using EMSA, that a protein present in crude extracts of $S$. coelicolor was able to delay the CRP-like cis-acting element found in the $c r p$ promoter [3]. However, EMSAs with purified $\mathrm{Crp}^{\mathrm{Sco}}{ }^{\mathrm{SHis}}{ }_{6}$ performed in the presence and absence of cAMP were unsuccessful. With the idea that the histidine tag adjacent to the DNA-binding domain might interfere with DNA binding, we established the purification of native $\mathrm{Crp}^{\mathrm{Sco}}$ produced in S. lividans and purified by cAMP affinity chromatography (Fig. 5). However, EMSAs performed with this protein, either with or without addition of cAMP, revealed the same result (Fig. 6A). We previously identified eight CRP-like cis-acting elements in the genome of $S$. coelicolor and presented them as possible target sites for $\mathrm{Crp}^{\mathrm{Sco}}$ [3]. So far, all tested CRP-like DNA sequences were unable to interact with $\mathrm{Crp}^{\mathrm{Sco}}$ (data not shown). The diversity of the conditions tested tends to indicate that $\mathrm{Crp}^{\mathrm{Sco}}$ either shows an important difficulty to bind by itself CRP-like cis-acting elements or that it is just required for the transcriptional activation of another transcription factor.

Other data further support that $\mathrm{Crp}^{\mathrm{Sco}}$ may not interact with the CRP-like site of its own promoter. In fact, in vivo complementation assays were performed to demonstrate whether $\mathrm{Crp}^{\mathrm{Sco}}$ was mechanistically similar to $\mathrm{Crp}^{\mathrm{Eco}}$, Vfr or GlxR regarding the recognition of CRP-like binding sites. We were unable to complement the maltose- and lactose-negative phenotype of an $E$. coli crp mutant in trans with $\mathrm{Crp}^{\text {Sco }}$ (Fig. 6B), although we could demonstrate by Western blot that $\mathrm{Crp}^{\mathrm{Sco}}$ was expressed (data not shown). In contrast, the cloned $g l x R$ or $v f r$ genes could complement a $E$. coli crp deletion mutant $[19,28]$. This unsuccessful complementation sug- 


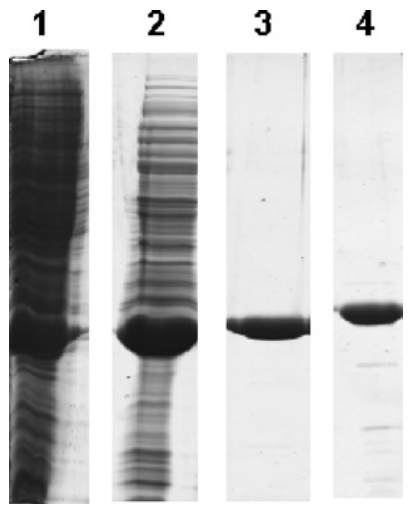

Fig. 5. cAMP-binding approach to purify overexpressed $\mathrm{Crp}^{\mathrm{Sco}}$ in $S$. lividans TK24. Fifteen percent SDS-PAGE with crude extract of $S$. lividans overexpressing $\mathrm{Crp}^{\mathrm{Sco}}$ (lane 1), the fraction containing $\mathrm{Crp}^{\mathrm{Sco}}$ after the first step of purification (ion exchange chromatography, lane 2), the purified $\mathrm{Crp}^{\mathrm{Sco}}$ protein after the cAMP affinity chromatography (lane 3), and $\mathrm{Crp}^{\mathrm{Sco}}-\mathrm{His}_{6}$ previously purified (lane 4). The scanning of the protein profiles showed that overexpressed $\mathrm{Crp}^{\mathrm{Sco}}$ represented $14 \%$ of the starting extract, $30 \%$ of the fraction was recovered after the ion exchange chromatography step, and $97 \%$ of the fraction was recovered after cAMP affinity chromatography. Under the conditions used, we collected $2 \mathrm{mg}$ of pure $\mathrm{Crp}^{\text {Sco }}$ from a $100 \mathrm{ml}$ culture.
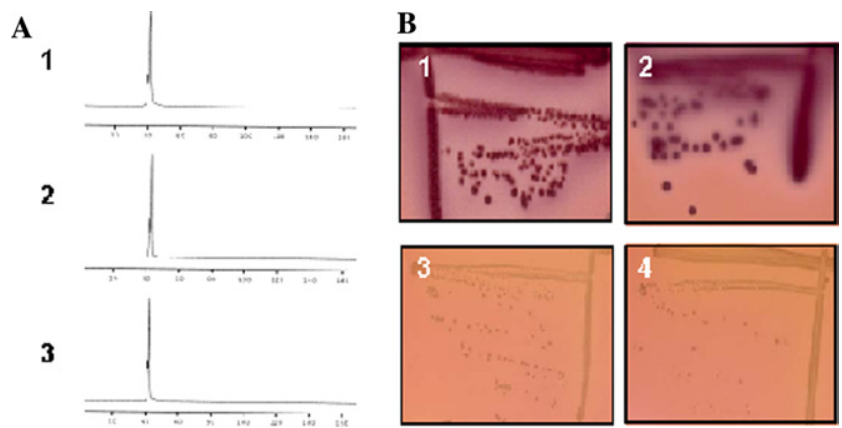

Fig. 6. DNA-binding inability of $\mathrm{Crp}^{\mathrm{Sco}}$ on the CRP-like cis-acting elements. (A) EMSA with $8 \times 10^{-6} \mathrm{M}$ purified $\mathrm{Crp}^{\text {Sco }}$ (lane 1, free DNA-probe; lane 2, DNA probe and pure $\mathrm{Crp}^{\mathrm{Sco}}$ without CAMP; and lane 3 , DNA probe and pure $\mathrm{Crp}^{\mathrm{Sco}}$ with $2 \times 10^{-2} \mathrm{M}$ cAMP). The time after detection of the fluorescent DNA-probe is indicated in minutes in the abscissa. (B) Complementation assay of the E. coli crp mutant LDN-3. The figure shows growth on MacConkey maltose plates, on which maltose-fermenting colonies appear red and maltose-negative colonies are white. 1, E. coli DH5 $\alpha$; 2, LDN-3(pFT172 $\left.c r p^{\mathrm{Eco}+}\right) ; 3$, LDN-3(pFT171 crp $^{\mathrm{Sco}+}$ ); and 4, LDN-3(pFT76 no crp gene present). (For interpretation of the references to color in this figure legend, the reader is referred to the web version of this paper).

gests that the way $\mathrm{Crp}^{\mathrm{Sco}}$ interacts with DNA should mechanistically differ from its most similar members and that the recognised cis-acting sequence should differ from the defined CRP-like consensus.

As in vitro and in vivo experiments suggested a specific DNA-binding ability for $\mathrm{Crp}^{\text {Sco }}$ we checked whether its modelised DNA-binding domain presented a $3 \mathrm{D}$ organisation similar to $\mathrm{Crp}^{\mathrm{Eco}}$. A superimposition of $\mathrm{Crp}^{\mathrm{ScO}}$ and $\mathrm{Crp}^{\mathrm{Eco}}$ was performed using only the backbone atoms of the residues composing the helix-

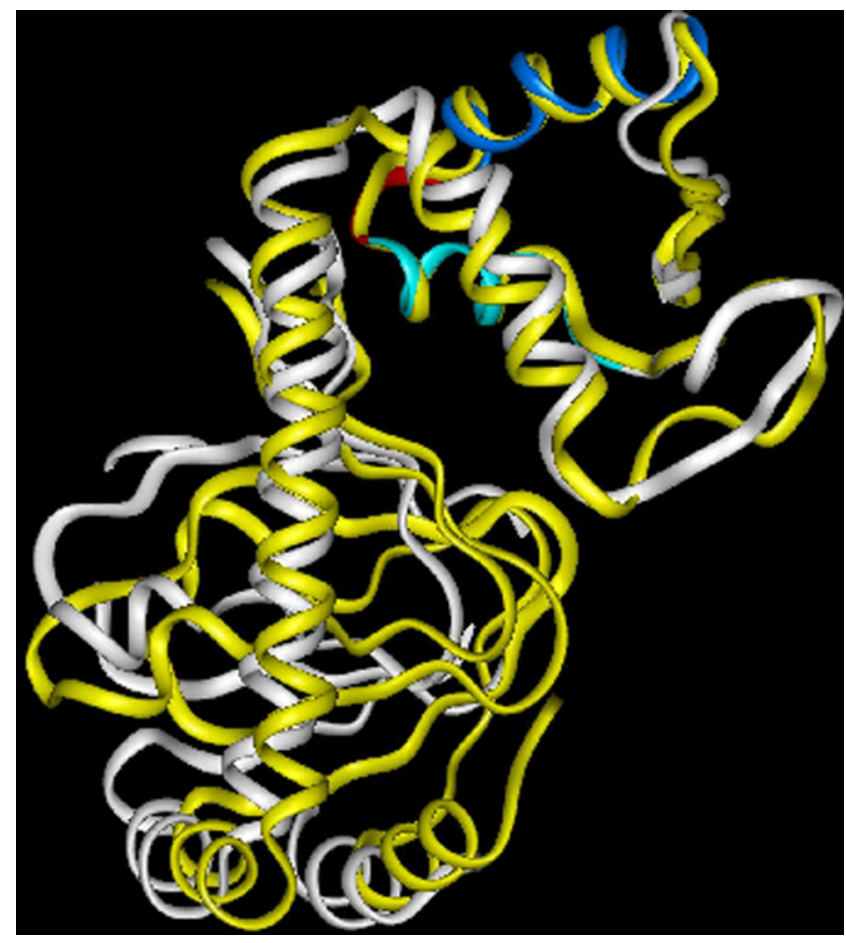

Fig. 7. Modelisation of the DNA-binding domain of $\mathrm{Crp}^{\mathrm{Sco}}$. Superimposition of the $\mathrm{Crp}^{\mathrm{Sco}}$ and $\mathrm{Crp}^{\mathrm{Eco}}$ in their optimised geometry. $\mathrm{C}_{\alpha}$ ribbon view of the $\mathrm{Crp}^{\mathrm{Sco}}$ (white) and $\mathrm{Crp}^{\mathrm{Eco}}$ (yellow) with the helixturn-helix motif highlighted in cyan-red-blue for Crp ${ }^{\mathrm{Sco}}$. (For interpretation of the references to color in this figure legend, the reader is referred to the web version of this paper.)

turn-helix (HTH) motif thought to be involved in DNA binding (Fig. 7). Apparently, these HTH motifs are fitting very well with one another but the relative position of the rest of the DNA-binding domain differs a lot from what is observed in $\mathrm{Crp}^{\mathrm{Eco}}$. Moreover, the average distance between the effector-binding domain and the DNA-binding domain is considerably increased in $\mathrm{Crp}^{\mathrm{Sco}}$ (data not shown). This positional rearrangement of the two domains must impose a different orientation and presentation of the HTH motif in the dimeric form, ultimately reflected by the accommodation of another cis-acting element as the CRP-like one.

The expression level of $\mathrm{Crp}^{\text {Sco }}$ being variable during the life cycle (data not shown) suggests that the cis-element of its promoter must be recognised by another regulatory protein. Such a putative candidate could be the Rex protein, a novel sensor of the NADH/NAD ${ }^{+}$redox poise [29]. Rex shares strikingly similar properties with those of Crp-like transcription factors although it does not belong to the CRP-FNR superfamily. In fact, Rex binds to the cis-elements of the cyd and nua operons, which possess the DNA palindromic pattern for CRP-FNR members. Furthermore, Brekasis and Paget [29] also showed that $\mathrm{NAD}^{+}$competes with NADH for Rex DNA binding, and it is now known that sites able to bind $\mathrm{NAD}^{+}$have also affinity for cAMP $[24$ 
26]. According to these relevant observations, EMSAs with purified Rex protein, with or without $\mathrm{Crp}^{\mathrm{Sco}}$ and cAMP as a putative partner(s) for cooperative binding, strongly need to be investigated on the CRP-like cis-acting element upstream crp.

\section{Conclusions}

The data reported in this work lead to four conclusions: (i) They correlate the previously obtained in vivo results of adenylate cyclase [4] and crp [3] null mutants strengthening the idea that cAMP is a key biomolecule responsible for correct spore germination in $S$. coelicolor, in which a Cya/cAMP/Crp system centrally triggers the developmental process. (ii) They demonstrate that $\mathrm{Crp}^{\mathrm{Sco}}$ is, after $\mathrm{Crp}^{\mathrm{Eco}}$ and $\mathrm{Vfr}$ of $P$. aeruginosa [17], the third member of the large CRP-FNR superfamily that binds cAMP. (iii) The cAMP-Crp ${ }^{\text {Sco }}$ interaction was strongly suggested from our refined in silico analysis that is based on a position-specific analysis, which appears to be more reliable than prediction based multiple alignments of full-length sequences as already reported for LacI-GalR members [30]. Based on our analysis, we predict that GlxR from C. glutamicum and homologues of mycobacterial and other actinomycetes species will soon join the CRP subgroup. (iv) Unsuccessful EMSAs and complementation assays as well as modelisation studies provide compelling evidence that $\mathrm{Crp}^{\mathrm{Sco}}$ is unable to bind CRP-like cis-acting elements. It remains to be demonstrated whether $\mathrm{Crp}^{\mathrm{Sco}}$ binds other $c i s$-acting elements or whether the predicted unusual three-dimensional feature of its DNA-binding domain impedes any DNA-protein interaction.

\section{Acknowledgments}

The Belgian government supported this work as part of the Interuniversity Poles of Attraction Programme (PAI P5/33) and through Grants SFB473 and GK805 of the Deutsche Forschungsgemeinschaft. A.D. and G.M. are research fellows of the Fund of Research in Industry and Agriculture (FRIA), and S.R. is a postdoc research assistant of the patrimony of the University of Liège. We thank Bernard Joris, Jean-Marie Frère, and Catherine Raskin for helpful discussions and Iris Thamm for kind assistance in DNA sequencing and gel shift assays.

\section{References}

[1] B.M.J. Kieser T., M.J. Buttner, K.F. Chater, D.A. Hopwood, Pratical Streptomyces Genetics, third ed., 2000. The John Innes Foundation, Norwich.
[2] D. Eaton, J.C. Ensign, Streptomyces viridochromogenes spore germination initiated by calcium ions, J. Bacteriol. 143 (1980) $377-382$.

[3] A. Derouaux, S. Halici, H. Nothaft, T. Neutelings, G. Moutzourelis, J. Dusart, F. Titgemeyer, S. Rigali, Deletion of a cyclic AMP receptor protein homologue diminishes germination and affects morphological development of Streptomyces coelicolor, J. Bacteriol. 186 (2004) 1893-1897.

[4] U. Susstrunk, J. Pidoux, S. Taubert, A. Ullmann, C.J. Thompson, Pleiotropic effects of cAMP on germination, antibiotic biosynthesis and morphological development in Streptomyces coelicolor, Mol. Microbiol. 30 (1998) 33-46.

[5] H. Korner, H.J. Sofia, W.G. Zumft, Phylogeny of the bacterial superfamily of Crp-Fnr transcription regulators: exploiting the metabolic spectrum by controlling alternative gene programs, FEMS Microbiol. Rev. 27 (2003) 559-592.

[6] J. Lengeler, A.M. Auburger, R. Mayer, A. Pecher, The phosphoenolpyruvate-dependent carbohydrate: phosphotransferase system enzymes II as chemoreceptors in chemotaxis of Escherichia coli K 12, Mol. Gen. Genet. 183 (1981) 163-170.

[7] D. Sabourn, J. Beckwith, Deletion of the Escherichia coli crp gene, J. Bacteriol. 122 (1975) 338-340.

[8] F. Vanrobaeys, B. Devreese, E. Lecocq, L. Rychlewski, L. De Smet, J. Van Beeumen, Proteomics of the dissimilatory ironreducing bacterium Shewanella oneidensis MR-1, using a matrixassisted laser desorption/ionization-tandem-time of flight mass spectrometer, Proteomics 3 (2003) 2249-2257.

[9] F. Corpet, Multiple sequence alignment with hierarchical clustering, Nucleic Acids Res. 16 (1988) 10881-10890.

[10] C. Lambert, N. Leonard, X. De Bolle, E. Depiereux, ESyPred3D: prediction of proteins 3D structures, Bioinformatics 18 (2002) $1250-1256$.

[11] P. Discover, Biosym Technologies, Inc., 9685 Scranton road, San Diego, CA 92121-2777, USA, 1993. Available from: $<$ www.accelrys.com $>$.

[12] S.J. Weiner, P.A. Kollman, AMBER: assisted model building with energy refinement. A general program for modeling molecules and their interactions, J. Comp. Chem. 2 (1981) 287303.

[13] S.J. Weiner, P.A. Kollman, D.T. Nguyen, D.A. Case, An all atom force field for simulations of proteins and nucleic acids, $\mathrm{J}$. Comp. Chem. 7 (1986) 230-252.

[14] C. Raskin, C. Gerard, S. Donfut, E. Giannotta, G. Van Driessche, J. Van Beeumen, J. Dusart, BlaB, a protein involved in the regulation of Streptomyces cacaoi beta-lactamases, is a penicillinbinding protein, Cell Mol. Life Sci. 60 (2003) 1460-1469.

[15] P. Filee, M. Delmarcelle, I. Thamm, B. Joris, Use of an ALFexpress DNA sequencer to analyze protein-nucleic acid interactions by band shift assay, Biotechniques 30 (2001) 1044 1048, 1050-1041.

[16] G. Zubay, D. Schwartz, J. Beckwith, Mechanism of activation of catabolite-sensitive genes: a positive control system, Proc. Natl. Acad. Sci. USA 66 (1970) 104-110.

[17] S.J. Suh, L.J. Runyen-Janecky, T.C. Maleniak, P. Hager, C.H. MacGregor, N.A. Zielinski-Mozny, P.V. Phibbs Jr., S.E. West, Effect of vfr mutation on global gene expression and catabolite repression control of Pseudomonas aeruginosa, Microbiology 148 (2002) 1561-1569.

[18] H. Youn, R.L. Kerby, G.P. Roberts, The role of the hydrophobic distal heme pocket of CooA in ligand sensing and response, J. Biol. Chem. 278 (2003) 2333-2340.

[19] H.J. Kim, T.H. Kim, Y. Kim, H.S. Lee, Identification and characterization of $g l x R$, a gene involved in regulation of glyoxylate bypass in Corynebacterium glutamicum, J. Bacteriol. 186 (2004) 3453-3460.

[20] S. Rigali, A. Derouaux, F. Giannotta, J. Dusart, Subdivision of the helix-turn-helix GntR family of bacterial regulators in the 
FadR, HutC, MocR, and YtrA subfamilies, J. Biol. Chem. 277 (2002) 12507-12515.

[21] M. Brodbeck, A. Rohling, W. Wohlleben, C.J. Thompson, U. Susstrunk, Nucleoside-diphosphate kinase from Streptomyces coelicolor, Eur. J. Biochem. 239 (1996) 208-213.

[22] M.S. Reddy, N. Guhan, K. Muniyappa, Characterization of single-stranded DNA-binding proteins from Mycobacteria. The carboxyl-terminal of domain of SSB is essential for stable association with its cognate RecA protein, J. Biol. Chem. 276 (2001) 45959-45968.

[23] P.W. Van Ophem, J. Van Beeumen, J.A. Duine, Nicotinoprotein [NAD(P)-containing] alcohol/aldehyde oxidoreductases. Purification and characterization of a novel type from Amycolatopsis methanolica, Eur. J. Biochem. 212 (1993) 819-826.

[24] K. Laukens, L. Roef, E. Witters, H. Slegers, H. Van Onckelen, Cyclic AMP affinity purification and ESI-QTOF MS-MS identification of cytosolic glyceraldehyde 3-phosphate dehydrogenase and two nucleoside diphosphate kinase isoforms from tobacco BY-2 cells, FEBS Lett. 508 (2001) 75-79.

[25] B. Weber, W. Weber, F. Buck, H. Hilz, Isolation of the myc transcription factor nucleoside diphosphate kinase and the multifunctional enzyme glyceraldehyde-3-phosphate dehydrogenase by cAMP affinity chromatography, Int. J. Biochem. Cell Biol. 27 (1995) 215-224.

[26] M.G. Rock, R.A. Cook, The effect of cyclic 3',5'-adenosine monophosphate on yeast glyceraldehyde-3-phosphate dehydrogenase. II. Initial velocity kinetic studies, Biochemistry 13 (1974) 4200-4204.

[27] A. Paiardini, R. Contestabile, S. D’Aguanno, S. Pascarella, F. Bossa, Threonine aldolase and alanine racemase: novel examples of convergent evolution in the superfamily of vitamin B6-dependent enzymes, Biochim. Biophys. Acta 1647 (2003) 214-219.

[28] S.E. West, A.K. Sample, L.J. Runyen-Janecky, The vfr gene product, required for Pseudomonas aeruginosa exotoxin A and protease production, belongs to the cyclic AMP receptor protein family, J. Bacteriol. 176 (1994) 7532-7542.

[29] D. Brekasis, M.S. Paget, A novel sensor of NADH/NAD ${ }^{+}$redox poise in Streptomyces coelicolor A3(2), Embo J. 22 (2003) 48564865.

[30] K. Fukami-Kobayashi, Y. Tateno, K. Nishikawa, Parallel evolution of ligand specificity between LacI/GalR family repressors and periplasmic sugar-binding proteins, Mol. Biol. Evol. 20 (2003) 267-277. 